\title{
Nano liquid chromatography columns
}

Steven Ray Wilson ${ }^{1,2}$, Christine Olsen ${ }^{1}$, Elsa Lundanes ${ }^{1 *}$

${ }^{1}$ Department of Chemistry, University of Oslo, Oslo, Norway

${ }^{2}$ Hybrid Technology Hub-Centre of Excellence, Institute of Basic Medical Sciences, Faculty of Medicine, University of Oslo, Oslo, Norway

Corresponding author: Professor Elsa Lundanes. E-mail: elsa.lundanes@kjemi.uio.no. Full address: Department of Chemistry, University of Oslo, Post Box 1033, Blindern, NO-0315 Oslo, Norway. Telephone: +4722855553

\begin{abstract}
Nano liquid chromatography (nanoLC), with columns having an inner diameter (ID) of $\leq 100 \mu \mathrm{m}$, can provide enhanced sensitivity and enable analysis of limited samples. NanoLC has become an established tool in omics research, and is gaining ground in other applications as well. There are several variants and formats of nanoLC columns, including packed columns, monoliths, open tubular columns, and the pillar array format. Most applications are done with packed columns, while e.g. the monolith and open tubular columns are still less established as routine tools. The pillar array format is a new variant with excellent resolution and low backpressure, and has recently been commercialized and used for bio-applications. In this minireview, we summarize and discuss recent research on nanoLC column development and uses, focusing on literature between 2016 and medio 2019.
\end{abstract}

Keywords: Packed column; Monolith; Open tubular; Pillar array; Chip, Extended-nano 


\section{Introduction}

Liquid chromatography (LC) is widely used for separating compounds prior to detection, and continues to be a key tool in analytical chemistry. However, the chromatographic process dilutes the analytes from injection to detection, and this can affect sensitivity, a central factor in the analysis of e.g. limited samples. A key factor in the dilution is the inner diameter (ID) of the column ${ }^{1}$. By reducing the column ID, the concentration of eluted compounds becomes higher, and enhanced sensitivity is obtained when coupled with concentration sensitive detectors like the electrospray ionization mass spectrometer (ESI-MS). Columns with reduced IDs include the microscale variant (about $1 \mathrm{~mm} \mathrm{ID),} \mathrm{the} \mathrm{capillary} \mathrm{LC}$ variant (about 0.2-0.5 $\mathrm{mm} \mathrm{ID}$ ), and the nanoLC variant, herein defined as a column with cylinder formed tube with ID $\leq 100 \mu \mathrm{m}$ or as a chip format column with $\leq 100 \mu \mathrm{m}$ channel depth/width. Columns with ID $\leq 100 \mu \mathrm{m}$ may also be included in the capillary column notation ${ }^{2}$. A recent review paper by Novotny gives a nice historical account on the development of capillary LC all the way to the nanoscale ${ }^{3}$. The nanoLC columns can in both formats (tube and chip) be filled with particles (particle packed) or with a porous continuous monolithic structure (monolith), or have an open structure (open tubular). In addition, pillar array columns can be used in the chip format ${ }^{4}$.

In this mini-review, we focus entirely on nanoLC due to its strong advantages related to sensitivity, but also, in many cases, chromatographic resolution. NanoLC in combination with MS is also a powerful combination, and has proven to be an immensely important tool for proteomics ${ }^{5}$. Also in metabolomics, nanoLC-MS has become an important technique because of its increased sensitivity relative to that obtained with larger ID columns ${ }^{6}$. Thus, much of the work described here also feature MS analysis. However, nanoLC is not limited to "omics", and has furthermore become of increasing interest for e.g. enantiomer separations ${ }^{7,8}$. Due to nanoLC's compatibility with limited sample amounts, emerging bio-applications include single-cell analyses and increasing focuses on clinical analysis.

In this minireview, we discuss the characteristics, recent developments, applications, obstacles and future opportunities of nanoLC columns in papers published from 2016 until medio 2019. The development of instrumentation is not included; instead, the reader is referred to reviews on miniaturized LC instrumentation ${ }^{9}$ and nanoLC platforms ${ }^{10}$. The small format and samples sizes of nanoLC can present challenges to the operator, often requiring add-ons such as online sample handling, but these issues have not been addressed in this review.

The authors reviewed the use of nano columns in proteomics in $2015^{1}$ and several other recent reviews also show the applicability of nanoLC $C^{5-7}$. Subjects of nanoLC we focus upon are: packed columns, monoliths, open tubular LC columns, pillar array columns, the chip format, and extended-nanoLC. 


\section{Packed columns}

Commercial columns with nanoLC dimensions and their application areas

At present, packed columns in the tube format are the most common columns in nanoLC. Packed columns are commercially available with inner diameter of 50 and $75 \mu \mathrm{m}$ from several vendors (e.g. Agilent, Thermo Fisher, Waters, Sigma Aldrich, Dr. Maisch). These columns are packed with particles of $1.7-3.5 \mu \mathrm{m}$, and come in various lengths, and typically with a C18 stationary phase. Very narrow ID columns (10-30 $\mu \mathrm{m})$, intended for ultra-sensitive analyses, are commercially available from at least one vendor (CoAnn Technologies), and packed with $1.7 \mu \mathrm{m}$ particles with C18 stationary phase.

The $75 \mu \mathrm{m}$ ID columns have become more or less a standard in proteomic analyses ${ }^{11-13}$, and also for the glycated proteome ${ }^{14}$. The $75 \mu \mathrm{m}$ ID C18 columns have also been applied in metabolomics ${ }^{15}$ and for the determination of drug of abuse ${ }^{16}$.

\section{New/in-house packed nanoLC columns}

Several research groups prefer to pack their own nanoLC columns for various reasons, e.g. cost and flexibility. Here we provide some examples of this approach. A $75 \mu \mathrm{m}$ ID column was in-house packed with $\mathrm{C} 18$ particles $(1.9 \mu \mathrm{m})$ and used by the group of $\mathrm{M}$. Mann for high coverage proteomics ${ }^{17}$, while Berg et al. in-house packed a $50 \mu \mathrm{m}$ ID column with $2.6 \mu \mathrm{m}$ core shell particles (C18 stationary phase) for targeted proteomics ${ }^{18}$. Spencer et al. used an in-house packed $75 \mu \mathrm{m}$ ID pulled tip analytical column with $3 \mu \mathrm{m} \mathrm{C18}$ particles in their automated trapping column exchanger system applied for proteomics $^{19}$. An in-house packed column (75 $\mu \mathrm{m}$ ID and $3 \mu \mathrm{m} \mathrm{C18}$ particles) was also used in a study where electron ionization MS was used for elucidation of the free fatty acid profile in mussel samples ${ }^{20}$. While the latter studies all used C18 stationary phases, an in-house made stationary phase was used for enantiomer separation in the nano format (75 $\mu \mathrm{m}$ ID) by D'Orazio et al. ${ }^{21}$ Use of packed columns with ID less than $50 \mu \mathrm{m}$ is not common. However, Shao et al recently packed $1.7 \mu \mathrm{m}$ C18 particles into a $22 \mu \mathrm{m}$ ID column, with a $3 \mu \mathrm{m}$ ID tip, for single-cell proteomics ${ }^{22}$ (Figure 1). A $70 \mathrm{~cm}$ long in-house packed PicoFrit column with $30 \mu \mathrm{m} I D$ and a tip size of $10 \mu \mathrm{m}$ was used in combination with a $4 \mathrm{~cm}$ long $100 \mu \mathrm{m}$ ID trap column, using $3 \mu \mathrm{m} \mathrm{C18} \mathrm{particles} \mathrm{in} \mathrm{both,} \mathrm{by} \mathrm{Zhu} \mathrm{et} \mathrm{al.} \mathrm{in} \mathrm{their} \mathrm{nanodroplet}$ processing platform for proteome profiling of $10-100$ mammalian cells ${ }^{23}$.

Figure 1 approximately here

Particles used in nanoLC columns 
Totally porous particles are still the most common in packed nanoLC columns, and most of the commercial columns are packed with silica-based totally porous particles. However, a few vendors (e.g. Thermo Scientific and Biotech) sell $75 \mu \mathrm{m}$ ID columns packed with silica-based superficially porous (core shell) particles. Totally porous particles were used in most of the studies mentioned above ${ }^{11-17,}$ ${ }^{19-22}$, and only one used core shell particles ${ }^{18}$. However, the benefits of core shell particles (e.g. high efficiency with reduced backpressure), and the fact that columns packed with such particles now are commercially available should increase their use in the future. For an overview of advantages of coreshell particles, see e.g. the review by Tanaka and McCalley and references therein ${ }^{24}$. Nonporous particles have also been used in the nanoLC column format. The group of Wirth has used submicrometer particles and found that the backpressure was reduced due to slip flow ${ }^{25}$, however, no recent studies using such particles has been published to the authors' knowledge.

\section{Sample introduction; trap column - analytical column combinations}

To maintain a high efficiency with direct-injection nanoLC, only a few $\mathrm{nLS}$ should be injected. Hence, sample introduction methods which allow for more of the sample to be introduced (=better chance of analyte detection) without being detrimental to the column performance is wanted. Indeed, such systems are widely used in nanoLC. Typically, a trap column, also called pre-column or solid phase extraction (SPE) column, is used, although not always ${ }^{16,20,22}$. The ID of the trap column is typically larger than that of the analytical column. As an example, Levernæs et al. used a $1 \mathrm{~mm} I D$ trap column in combination with a $75 \mu \mathrm{m}$ ID analytical column ${ }^{12}$. To minimize effects of void volumes, the trap column may be packed with larger particles or a material providing less retention relative to that on the analytical column in order to have some phase focusing and thus improved efficiency. Zhang et al. used a $300 \mu \mathrm{m}$ ID trap column packed with $5 \mu \mathrm{m}$ C18 particles in combination with a $75 \mu \mathrm{m}$ ID column packed with $2 \mu \mathrm{m}$ C18 particles ${ }^{14}$. In addition, they used an in-house packed boronate affinity enrichment column ( $1 \mathrm{~mm}$ ID) upstream the $\mathrm{C} 18$ trap column in an online system for glycated peptides. The trap column and the analytical column had the same ID $(75 \mu \mathrm{m})$, but the trap column was packed with larger particles ( $3 \mu \mathrm{m}$ vs. $2 \mu \mathrm{m}$ ) in a quantitative metabolomics study ${ }^{15}$. Berg et al. found that a C8 monolithic trap column performed better than a standard C18 packed trap column (both $50 \mu \mathrm{m} \mathrm{ID} \mathrm{and}$ the same as ID of the analytical column) for targeted proteomics ${ }^{18}$. Schöbinger et al. ${ }^{11}$, carrying out the separation at $\geq 45{ }^{\circ} \mathrm{C}$, pointed out that loading the sample at high temperature may cause loss of sample. They used a low-temperature mobile phase for trapping of peptides on a $300 \mu \mathrm{m}$ ID trap column packed with $5 \mu \mathrm{m}$ C18 particles, in combination with a $75 \mu \mathrm{m}$ ID analytical column packed with $3 \mu \mathrm{m}$ C18 particles.

Another sample introduction system is the "speLC" system described by Falkenby et $\mathrm{al}^{26}$. The speLC uses C18 StageTips (small C18-based SPE microcolumns with a peptide binding capacity of up to $5 \mu \mathrm{g}$ ). 
Instead of eluting fractions into the autosampler vial of the LC system, a low-pressure pump passes a 5-10 min gradient through the StageTip and then to the analytical column. The speLC-system eliminates sample-to-sample carry-over by using disposable StageTips. In a recent paper, the same authors have improved their approach by capturing the analytes from the StageTip into a long capillary loop with a pre-formed gradient, allowing subsequent isocratic pump operation for the analytical column ${ }^{27}$. The authors claim that this system, which is now commercialized (Evosep One), provides sensitivity, throughput and robustness, and is applicable for large clinical studies.

Another approach to reduce sample-to-sample carry-over and increase throughput is that by Spencer et $\mathrm{al}^{19}$. They developed a trap column-exchanging robot that was equipped with four in-house packed $150 \mu \mathrm{m}$ ID trap columns, packed with $4 \mu \mathrm{m} \mathrm{C12}$ particles. They claim that the retention time was sufficiently repeatable using the four different trap columns without the need for rescheduling the selection windows, as long as the columns were repeatable packed.

To achieve high coverage proteomics another novel approach (called "spider fractionator") has been introduced ${ }^{17}$. In this approach, fractions from a $250 \mu \mathrm{m} \mathrm{ID} \mathrm{and} 30 \mathrm{~cm}$ long column packed with $1.9 \mu \mathrm{m}$ C18 particles and used with a high pH mobile phase, were transferred to the $75 \mu \mathrm{m}$ ID analytical column also packed with $1.9 \mu \mathrm{m}$ C18 particles (Figure 2).

In summary, packed columns are still the workhorse of nanoLC, with fully-porous particles dominating applications, but alternatives such as core shell materials are emerging along with decreasing particle sizes. Sample introduction techniques with these familiar materials continue to be developed. Key advantages include robustness and commercial availability. Key disadvantages include limitations on resolution related to back-pressure constraints and difficulties in particle-packing very narrow columns.

Figure2 approximately here

\section{Monolithic columns}

Monolithic columns can be categorized into three main types depending on their composition; organic polymer-based, silica-based and organic-silica hybrid monolith ${ }^{28}$.

\section{Commercial nanoLC monoliths}

While no longer the case regarding packed nanoLC columns, the number of commercial monolithic nanoLC columns is very limited ${ }^{29}$. Apparently, only one vendor produces (50 and $100 \mu \mathrm{m}$ ID) polymer- 
based monoliths, and two produce (50 and $100 \mu \mathrm{m}$ ID) silica-based monoliths. The PepSwift ${ }^{\mathrm{TM}}$ phase intended for peptide separations is based on a poly(divinylbenzene-coethylvinyl-benzene-styrene) copolymer. The ProSwift RP- $4 \mathrm{H}^{\mathrm{TM}}$ phase intended for intact protein separations is based on a poly(divinylbenzene-co-ethylvinylbenzene) co-polymer with similar selectivity as the PepSwift ${ }^{\mathrm{TM}}$ phase. The ProSwift C4 RP-5H ${ }^{\mathrm{TM}}$ phase possesses butyl functionality based on a poly(ethylene dimethacrylateco-butylmethacrylate) co-polymer providing a less hydrophobic selectivity.

The silica-based Chromolith $^{\circledR}$ CapRod $^{\circledR}$ nanoLC columns have a C18 stationary phase, but is also available with a C8 stationary phase, and in a selection of internal diameters $(50 \mu \mathrm{m}, 100 \mu \mathrm{m}$ and 200 $\mu \mathrm{m})$, pore structures (standard and high resolution), and lengths $(5,15$ and $30 \mathrm{~cm})$.

The MonoCap HighResolution 2000 is a $0.1 \mathrm{~mm}$ x 2 m length monolithic silica capillary column (from GL Science) with C18 or HILIC stationary phase designed for identifying an extremely high number of peptides/proteins for proteome research via LC-MS/MS. In a study comparing commercial nanoLC columns for fast, targeted proteomics, Vehus et al. found that the monolithic columns were more prone to retention time instability than packed columns ${ }^{30}$.

\section{Applications of monolithic nanoLC columns}

The extent to which these commercially available columns are used in applications, is not easily found searching for monolithic nanoLC in the literature. However, Kucera et al. reported the use of a $100 \mu \mathrm{m}$ ID Chromolith CapRod in a comparison of nanoLC and conventional LC for diastereoisomer separation $^{31}$. A $100 \mu \mathrm{m}$ ID silica-based C18 monolithic column identical to the Chromlith CapRod was used by Brandtzaeg et al. for detecting ricin $^{32}$ (Figure 3 ). A recent review paper on the use of polymerbased monolithic capillary columns and their applications in food analysis did not include studies using commercial columns ${ }^{33}$.

Figure 3 approximately here

\section{New monolithic nanoLC columns}

While there are few commercial nanoLC monolithic columns, a great number of columns with various chemistries have been developed in later years. Several recent reviews can be found on advances in the preparation of monolithic columns in the narrow format i.e. $\leq 100 \mu \mathrm{m} \mathrm{ID}{ }^{34-38}$. The current review includes papers published since 2016, and in the following new organic polymer-based, silica-based, as well as hybrid monoliths are included.

Organic polymer monoliths: Organic-based monoliths have a long history ${ }^{39}$, and are considered to be rather simple to make, as opposed to their silica-based sibling. An overview of the state-of-the-art and 
guidelines in tuning the macropore structure of polymer-based monoliths can be found in a recent review by Dores-Sousa et al ${ }^{37}$. Two papers from 2016 describe the trends in the development of porous polymer monoliths ${ }^{39,40}$. The research group of Hanfa Zou has used click polymerization for making both organic monoliths and organic-silica hybrids, and state in their review from 2016 that the efficiency of these is greatly improved compared with organic monoliths prepared by free radical polymerization ${ }^{41}$, which is more commonly used. Table 1 gives an overview of the most recent advances in organic polymer monolithic columns.

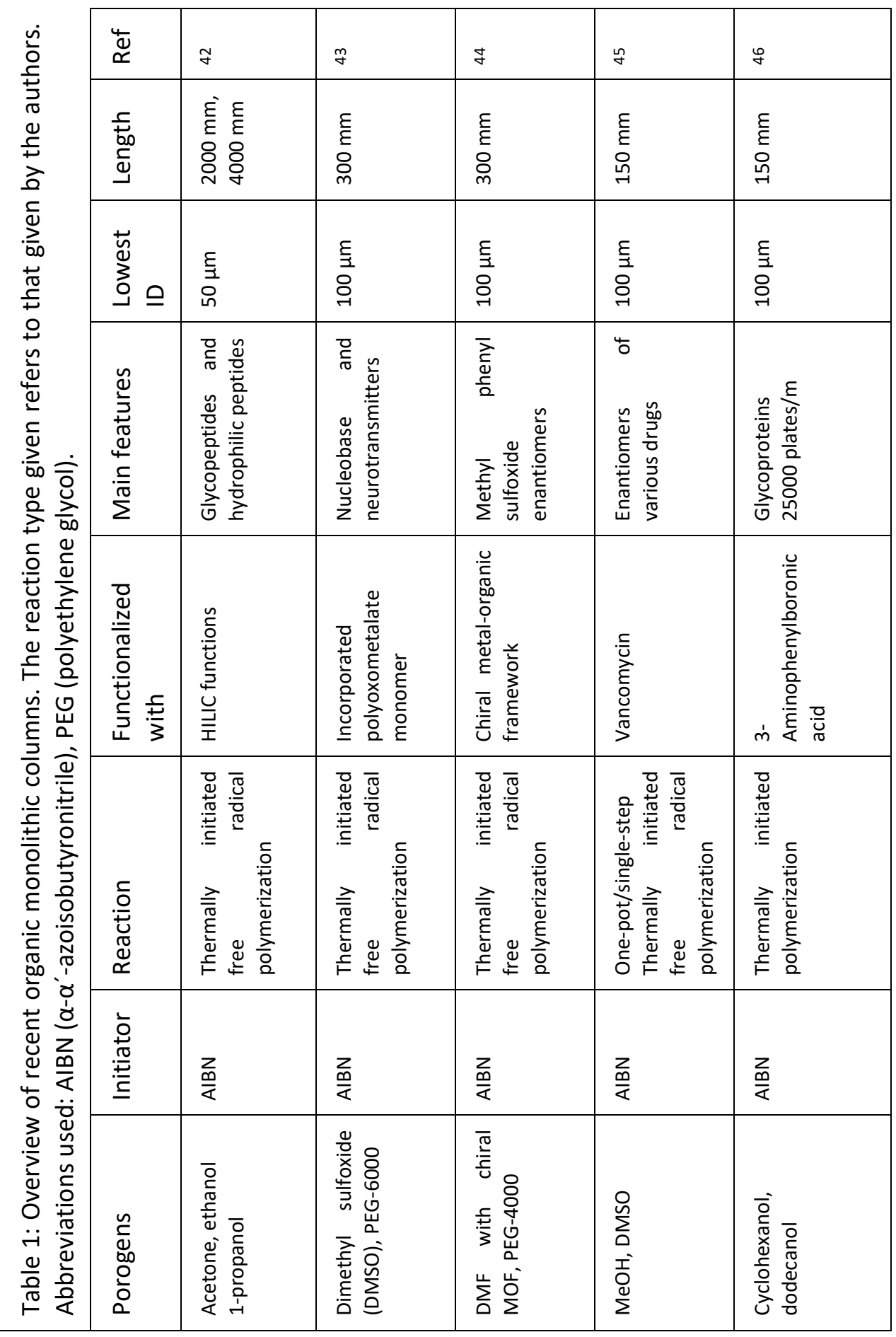




\begin{tabular}{|c|c|c|c|c|c|}
\hline $\begin{array}{l}\frac{n}{d} \\
\varepsilon \\
\frac{0}{0} \\
\stackrel{0}{\Sigma}\end{array}$ & 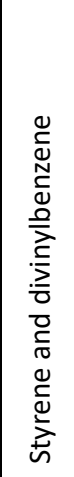 & 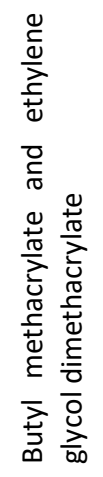 & 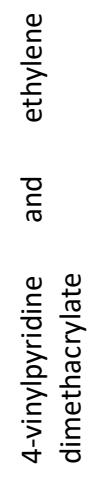 & 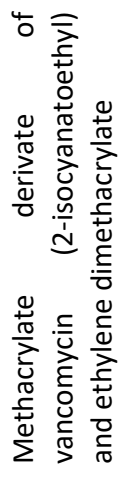 & 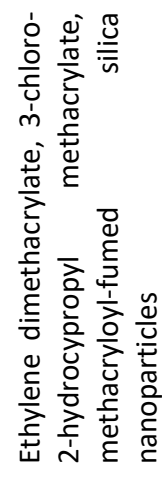 \\
\hline
\end{tabular}

Silica-based monoliths and stationary phases: The second-generation, with increased structural homogeneity relative to the first-generation ${ }^{47}$, monolithic silica-based columns in the capillary format can provide an efficiency which is comparable to that of a column packed with $2-2.5 \mu \mathrm{m}$ fully porous particles, while having a pressure drop comparable to that of a column packed with $5 \mu \mathrm{m}$ particles ${ }^{48}$. Hara et al. have actively contributed to the further development of silica-based monolithic capillary columns during the last three years ${ }^{48-50}$. The polymerization solution used for preparation of the monolithic silica-based columns consisted typically of $5.6 \mathrm{~mL}$ tetramethoxysilane (TMOS), $0.900 \mathrm{~g}$ urea, $10 \mathrm{~mL}$ of $0.01 \mathrm{M}$ aqueous acidic acid and $1.25-1.4 \mathrm{~g}$ of polyethyleneglycol (PEG) in a study on the effect of PEG on pore structure and separation efficiency ${ }^{48}$. Functionalization of the monolithic surface, after gelation, hydrothermal treatment and calcination, was done with an octadecylsilylation reagent. They found that the $100 \mu \mathrm{m}$ ID column prepared with $20,000 \mathrm{~g} / \mathrm{mol}$ PEG gave a plate height $\mathrm{H}=4.0$ $\mu \mathrm{m}$, which was the best obtained at that time for a monolithic column. Monolithic columns of length $25 \mathrm{~cm}$, and $50 \mu \mathrm{m}$ and $100 \mu \mathrm{m} \mathrm{ID}$, prepared using $5.6 \mathrm{~mL}$ TMOS, $0.09 \mathrm{~g}$ urea, $10 \mathrm{~mL} 0.01 \mathrm{M}$ aqueous acetic acid and $1.4 \mathrm{~g}$ of $20.000 \mathrm{~g} / \mathrm{mol}$ PEG, were found to be pressure stable up to the maximal test pressure of $80 \mathrm{MPa}^{49}$. Hara et al. also have made $5 \mu \mathrm{m}$ ID and $10 \mu \mathrm{m}$ ID TMOS-based silica-based monoliths with $\mathrm{C} 18$ stationary phase $\mathrm{s}^{50}$. These columns had very low capillary-to-domain size aspectsratios, including the absolute minimal aspect-ratio with only one single node in the cross-section. Their observations confirmed the classic observation in the past for packed columns (by Knox, Jorgenson and Kennedy) that ultralow aspect-ratio columns generate a markedly lower dispersion than larger aspect-ratio columns $s^{50}$. The lowest capillary-to-domain size aspect-ratio column had the best performance, but was still inferior to the open-tubular format. Kobayashi et al. made a $100 \mu \mathrm{m}$ ID silica monolith according to a prior reported procedure and used octadecyltrimethoxy silane to prepare a low-density octadecyl (ODS) monolith which was additionally modified, to study the effect of acidic mobile phase additives for peptide separations ${ }^{51}$. They found both high peak capacity and sensitivity using cyanoacetic acid as mobile phase additive with these phases.

Hybrid monoliths: It has been argued that silica-based monoliths are both tedious to prepare and have low chemical and mechanical strength, and this has been the reason for investigating other approaches 
to obtain high efficiency monolithic columns, such as organosilicon-based hybrid columns. A review published in 2017 covers the advances in organic-silica hybrid monoliths up to mid $2016^{28}$.

In Table 2 we present some recent studies focusing on hybrids. Most of the columns have been made using an one-pot (one-step), either photo-initiated or thermally initiated, polymerization.

\begin{tabular}{|c|c|c|c|c|c|c|c|c|c|c|c|c|c|c|c|c|c|}
\hline$\stackrel{\mathscr{D}}{\stackrel{\Phi}{ \pm}}$ & $\underset{\mathbb{\Sigma}}{\mathbb{\Sigma}}$ & $\tilde{n}$ & n̊ & मे & 另 & in & in & $\stackrel{\infty}{\sim}$ & & ด & 8 & $\vec{b}$ & $\widetilde{\sigma}$ & $\ddot{0}$ & d & นٌ & $\mathscr{\bullet}$ \\
\hline 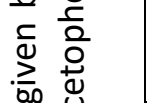 & $\begin{array}{l}\text { 歖 } \\
\underline{\underline{\underline{w}}}\end{array}$ & 尧 & 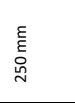 & 䲻 & 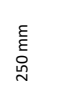 & 高 & 言 & 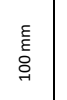 & 言 & 衰 & 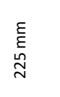 & 言 & $\begin{array}{l}\frac{\varepsilon}{\xi} \\
\stackrel{g}{g}\end{array}$ & 镸 & 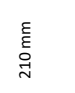 & 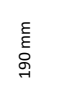 & 䲻 \\
\hline 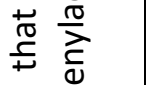 & $\varrho$ & 镸 & 镸 & 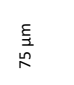 & 衰 & 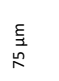 & 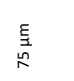 & 镸 & 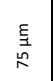 & 惡 & 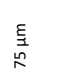 & 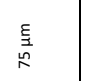 & 言 & 言 & 衰 & 惡 & 章 \\
\hline 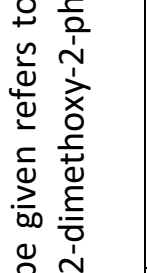 & 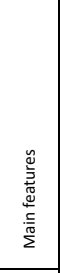 & 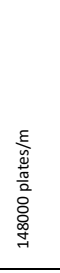 & 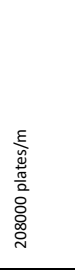 & 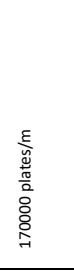 & 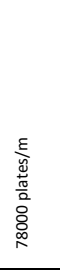 & 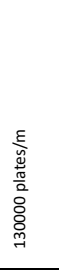 & 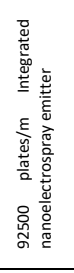 & 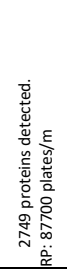 & & 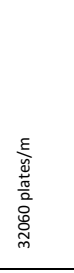 & 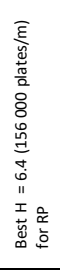 & 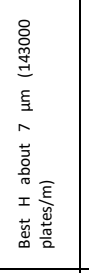 & 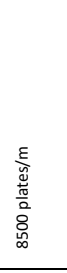 & 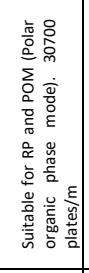 & 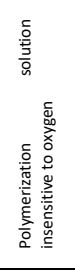 & 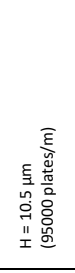 & 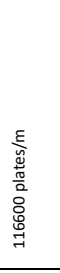 \\
\hline 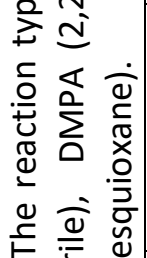 & 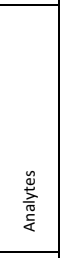 & 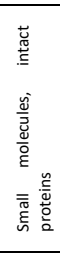 & 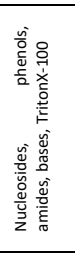 & 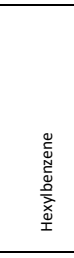 & 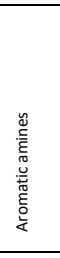 & 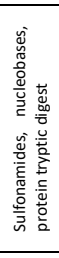 & 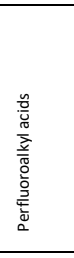 & 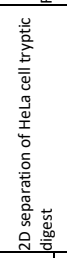 & & 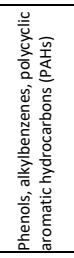 & 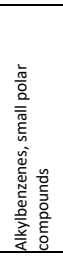 & 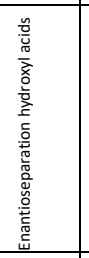 & $\frac{\delta}{\partial}$ & 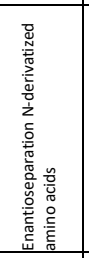 & 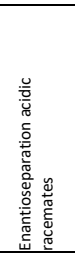 & 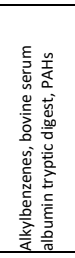 & 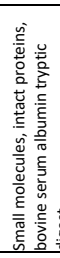 \\
\hline 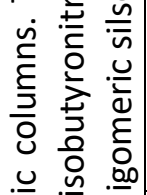 & 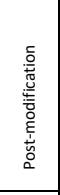 & 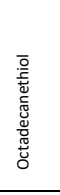 & 旁 & 营 & 旁 & 旁 & 旁 & 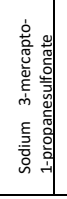 & 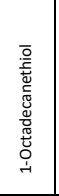 & 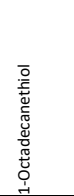 & 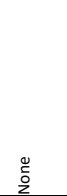 & 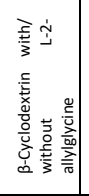 & $\stackrel{\frac{0}{5}}{\frac{0}{2}}$ & 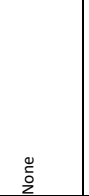 & $\frac{0}{\frac{0}{2}}$ & 空 & 突 \\
\hline 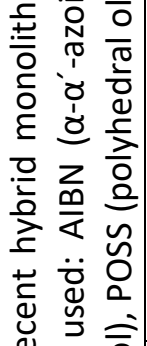 & 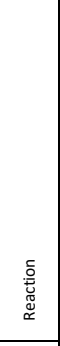 & 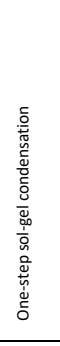 & 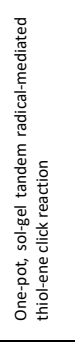 & 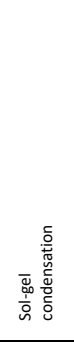 & 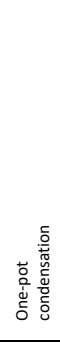 & 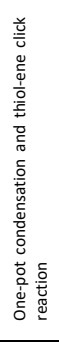 & 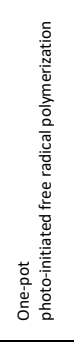 & 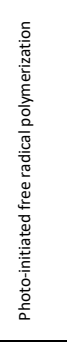 & & 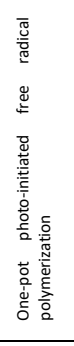 & 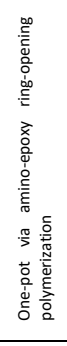 & 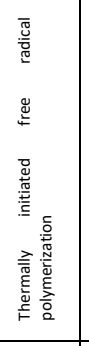 & 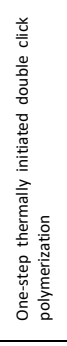 & 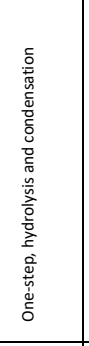 & 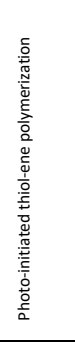 & 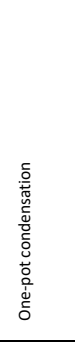 & 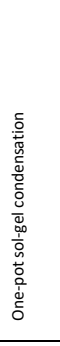 \\
\hline 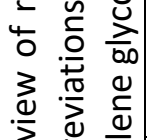 & 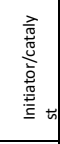 & 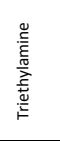 & 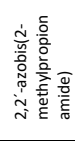 & 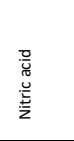 & 嫒 & 尊 & 產 & 亮 & & 產 & $\stackrel{\text { go }}{\frac{0}{2}}$ & 酸 & 䨗 & 畺 & $\stackrel{\frac{0}{5}}{\frac{0}{2}}$ & 尊 & 旁 \\
\hline 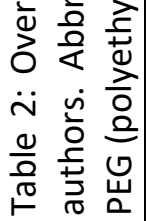 & 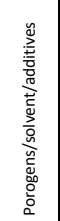 & 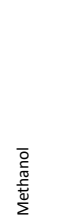 & 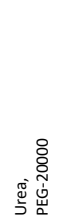 & 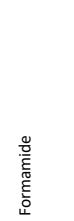 & 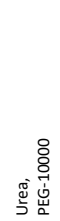 & : & 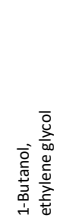 & 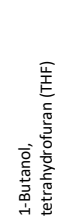 & & 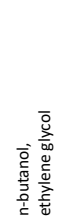 & 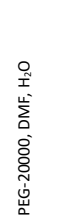 & 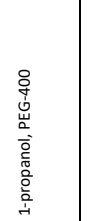 & 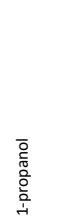 & 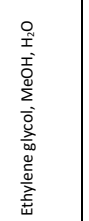 & 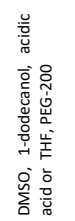 & 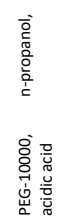 & 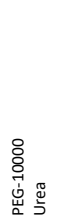 \\
\hline
\end{tabular}




\begin{tabular}{|c|c|c|c|c|c|c|c|c|c|c|c|c|c|c|c|}
\hline & 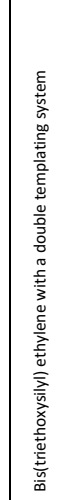 & 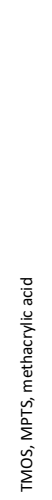 & 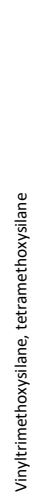 & 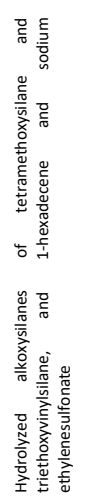 & 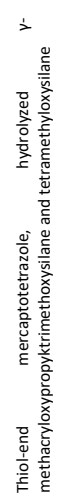 & 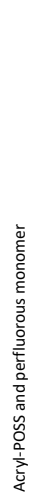 & 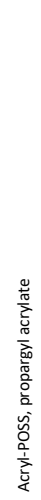 & 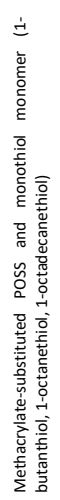 & 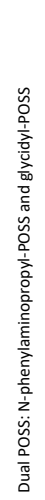 & 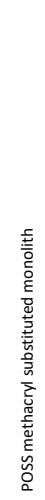 & 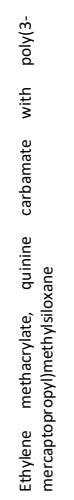 & 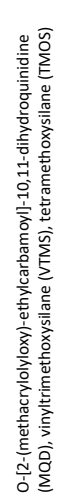 & 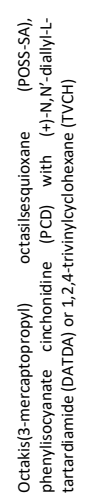 & 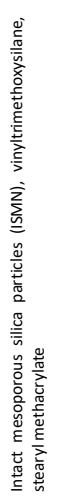 & 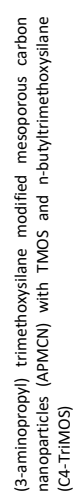 \\
\hline
\end{tabular}

\section{Sample introduction; trap column - analytical column combinations}

The non-commercial monolithic nanoLC columns described in the papers above have mostly been used for separation of standard mixtures. However, such monolithic columns may have been used for applications without being revealed in a literature search. One example of this is the paper on measurement of glycated albumin in serum and plasma by Brede et al. who used a $500 \mathrm{~mm}$ long and $20 \mu \mathrm{m}$ ID porous polymer monolith prepared in-house according to a patented procedure ${ }^{67}$. They used a trap column of the same ID, but $100 \mathrm{~mm}$ long, and injected $1 \mu \mathrm{L}$ of sample. As another example, and mentioned above; perfluoroalkyl acids in water samples were determined using a hybrid fluorous monolithic column ${ }^{57}$. The limit of quantification (LOQ) was $0.5 \mathrm{ng} / \mathrm{mL}$ using an injection volume of 0.44 $\mu \mathrm{L}$.

The recent review paper on the use of monolithic columns for intact proteins ${ }^{68}$, also shows that mostly standards and not real biological samples have been chromatographed. In a review by Shibasaki et al. on the molecular and physiological study of Candida albicans they refer to a study where an in-house made $470 \mathrm{~cm}$ long and $100 \mu \mathrm{m}$ ID C18 silica-based monolith was used ${ }^{69}$.

In summary, there is undoubtedly a high activity in the field of monolithic separation column development for nanoLC, with a large variety of selectivities. However, monolithic columns do not appear to be widely used in modern applications, as opposed to packed nanoLC columns. Key advantages of monolithic columns include low back pressures that allow for fast separations/long column separations. Key disadvantages include difficulties in reproducibility and commercial availability. 


\section{Open tubular columns}

Due to their theoretical high efficiency and small sample volume consumption open tubular (OT) columns have been a topic in LC since the late seventies ${ }^{70}$, however, the increased interest in such columns in the last decade stems from the work of Karger's group. They were the first to show the great potential of OT columns using a $10 \mu \mathrm{m}$ ID column with a porous layer of poly(styrene-codivinylbenzene) for proteomics ${ }^{71}$. Similar columns were applied in studies of stem cell-like cancer cells by the Lundanes group ${ }^{72,73}$. However, despite the advances in open tubular column LC, as reviewed recently by Lam et $\mathrm{al}^{74}$, to the authors' knowledge, such columns are not commercially available, yet.

\section{New OT columns with ID $\leq 10 \mu \mathrm{m}$}

From the papers published since 2016 on OT columns with ID $\leq 100 \mu \mathrm{m}$, there are two main directions; smaller ID columns, and the exploration of selective phases in the somewhat larger ID ( $25 \mu \mathrm{m}$ and larger) open format. Little has apparently been done on the development of poly(styrene-codivinylbenzene) OT columns, although columns with different layer thickness were made and characterized by Skjaervoe et $\mathrm{al}^{75}$. More focus has recently been on silica layer OT columns. Vehus et al. showed the potential for such columns for separation of small molecules, peptides and intact proteins ${ }^{76}$. However, the group of Desmet has been the most active in pursuing very high efficiency porous layer OT columns, which are produced via a sol-gel process ${ }^{77-79}$. They have made very high efficiency $5 \mu \mathrm{m}$ ID OT columns which gave up to 600000 plates on a $2.5 \mathrm{~m}$ long column with a $300 \mathrm{~nm}$ thick layer porous layer ${ }^{77}$. They used in-column sol-gel synthesis with a solution consisting of TMOS, urea, PEG, and aqueous acetic acid, with subsequent octadecylsilylation to obtain the reversed phase C18 stationary phase.

The injection was carried out using split-flow injection, and detection by on-column fluorescence detection using a confocal microscope. The mesopore size, and hence surface area, could be controlled by the temperature used during the hydrothermal treatment ${ }^{78}$. Recently they reported increased hydrophobicity of $5 \mu \mathrm{m}$ ID silica-based OT columns by applying hybrid TMOS/methyltrimethoxysilane (MTMS) layers with inserted methyl groups. Due to higher hydrophobicity, thinner porous layers gave similar retention factor $(k)$ as in octadecylsilylated columns synthesized using TMOS only. Since thinner layers have a lower intra-layer mass transfer resistance, superior column efficiencies were obtained compared to that of TMOS-based porous layer OT columns giving the same retention ${ }^{79}$. These columns obviously have a great potential for use in applications where high resolution is needed for samples of limited amount.

OT columns with $2 \mu \mathrm{m}$ ID have been prepared by Chen et al. ${ }^{80}$, and Yang et al. ${ }^{81}$ Chen et al. obtained plate heights of less than $0.1 \mu \mathrm{m}$ in less than $10 \mathrm{~min}$ and under an elution pressure of $c a .20$ bar using 
a trimethoxy(octadecyl)silane coated $2 \mu \mathrm{m}$ ID capillary. Yang et al. also used a $2 \mu \mathrm{m}$ ID OT column with the wall derivatized with trimethoxy(octadecyl) silane. Laser-induced fluorescence on-column detection was performed for analytes labeled with ATTO-TAG FQ, and 440 apparent peaks with a peak capacity of 1640 within 172 min were observed when separating a sample from pepsin/trypsindigested Escherichia coli cell lysate.

An even more narrow ID OT column is reported by Li et al. in their pico_LC system with a $0.9 \mu \mathrm{m}$ ID porous layer OT column with an integrated femtopipette. They used this system for separation of amino acid enantiomers ${ }^{82}$. The poly(MQD-co-HEMA-co-EDMA) picoporous layer OT column was prepared using an in situ thermal initiation polymerization method.

The fact that fluorescence detection has been used in the latter papers, illustrates the challenges with detection when the columns are becoming very narrow. The ID of the commercial nanospray emitters is not compatible with the narrow ID columns. In order to maintain the high efficiency of the narrow ID open tubular format, but having larger sample capacity and ease MS detection, Paull and coworkers have explored the use of multichannel (multilumen) capillaries ${ }^{83}, 84$. They used photonic crystal fibre which contained 126 internal parallel $4 \mu \mathrm{m}$ channels, with a porous poly(styrene-co-divinylbenzene) layer, and report run-to-run retention time repeatability below $1 \%^{83}$. In the more recent paper ${ }^{84}$, the same group reports a multi-lumen capillary (also with 126 parallel channels of $4.2 \mu \mathrm{m}$ ID) with a C18functionalised silica porous layer OT column for both on-capillary preconcentration and separation, followed by MS detection. Following modification, $100 \%$ of the channels displayed a homogenous porous silica layer, $257 \pm 36 \mathrm{~nm}$ thick. They state that the multi-channel structure allowed the capillary to be applied at higher flow rates which simplifies system requirements and increases detection options, however, the separation efficiencies could be improved. The possibility of using a multichannel capillary for increased sample loading has also been explored by Ribeiro da Silva et al. ${ }^{85}$, who used a multichannel capillary with 126 parallel channels of $8 \mu \mathrm{m}$ each as trap column. The channels were coated with a layer of poly(styrene-co-octadecene-co-divinylbenzene) (PS-OD-DVB). The trap column was coupled online with a $10 \mu \mathrm{m} \times 2 \mathrm{~m}$ poly(styrene-co-divinylbenzene) (PS-DVB) OT LC column with nanospray mass spectrometry detection. Compared to using monolithic/particlepacked trap columns, the multichannel OT trap column allowed both fast loading and sufficient refocusing of small model compounds (sulfonamides $\approx 300 \mathrm{Da}$ ) on the OT analytical column.

The challenge of introducing a sufficient sample volume in order to be able to detect analytes present at low concentrations in a sample, may be one of the reasons for the lack of applications reported using narrow ID OT columns. Vehus et al. used a high sample capacity $50 \mu \mathrm{m} \times 40 \mathrm{~mm}$ (PS-OD-DVB) monolithic trap column with sample capacity ( $>2000 \mathrm{ng}$ on a $10 \mathrm{~cm}$ column; comparable to the capacity of commercial particle-packed columns) in combination with a $10 \mu \mathrm{m} \times 3000 \mathrm{~mm}$ OT 
column functionalized with octadecyl groups bound to a silica skeleton that coats the wall of the column ${ }^{76}$. The approach used by Rodriguez et al. ${ }^{84}$, and Ribeiro da Silva et al. ${ }^{85}$ may also be a solution to look into.

\section{OT columns with larger ID}

Even though inferior efficiency is expected, several papers report the use of larger ID capillaries to prepare columns with various chemistries to obtain the desired selectivity. Peng et al. made and evaluated a $3 \mathrm{~m}$ long $25 \mu \mathrm{m}$ ID zwitterionic OT column with a $4 \mu \mathrm{m}$ porous layer, by copolymerization of [2-(methacryloyloxy)ethyl] dimethyl-(3-sulfopropyl) ammonium hydroxide and $N, N^{\prime}$ methylenebis(acrylamide). Separation of neutral, basic, and acidic compounds demonstrated the strong hydrophilicity of the stationary phase ${ }^{86}$. Wang et al. prepared a $25 \mu \mathrm{m}$ ID OT column by in situ ring-opening polymerization of octaglycidyldimethylsilyl polyhedral oligomeric silsesquioxanes (POSSepoxy) with 4-aminophenyl disulfide (APDS) in a binary porogenic system of ethanol/ $\mathrm{H}_{2} \mathrm{O}^{87}$. They found that the porogenic composition played an important role in the formation of OT stationary phases as the ratio of ethanol/ $\mathrm{H}_{2} \mathrm{O}$ at $6 / 1(\mathrm{v} / \mathrm{v})$ would lead to a hybrid monolith, while the ratio of ethanol/ $\mathrm{H}_{2} \mathrm{O}$ at $13 / 1(\mathrm{v} / \mathrm{v})$ resulted in OT phases. However, the efficiency of the column, evaluated by alkylbenzenes, was rather low. A tryptic digest of mouse liver proteins was used to evaluate the performance of a 2.5 $\mathrm{m}$ long OT column demonstrating the potential of such columns in proteome analysis.

Several columns have been prepared with metal-organic frameworks (MOFs) as stationary phases. Chen et al. prepared and evaluated a $25 \mu \mathrm{m}$ ID NH $\mathrm{N}_{2}$-UiO-66-modified pGMA OT column by modifying $\mathrm{NH}_{2}$-UiO-66 nanoparticles on the brush-shaped pGMA layer on the inner wall of the capillary ${ }^{88}$. Brushshaped pGMA can increase the bonding amount of the $\mathrm{NH}_{2}$-UiO- 66 with good column permeability. $\mathrm{NH}_{2}$-UiO-66 possesses a 3D structure, and the aromatic rings and amino groups in ligands can have hydrophobic interactions and hydrogen bonding interactions with analytes. The $\mathrm{NH}_{2}$-UiO-66-modified pGMA column was successfully applied for separation of small molecules with excellent column efficiency (121 500 plates on a $1.12 \mathrm{~m}$ long column) and selectivity, according to the authors. Zhu et al. also prepared and evaluated a MOF modified OT column ${ }^{89}$. A $25 \mu \mathrm{m}$ ID x 1m column was prepared by incorporating MOF particles modified with vancomycin directly into a zwitterionic polymer coating synthesized by the copolymerization of [2 (methacryloyloxy)ethyl]dimethyl-(3-sulfopropyl)ammonium hydroxide and N,N'-methylenebisacrylamide. The incorporation of IRMOF-3 (isoreticular MOF-3) particles improved selectivity of the zwitterionic polymer coating with absolute column efficiency reaching 79900 plates for $p$-xylene. Shao and Zhang designed a $20 \mu \mathrm{m}$ ID $\times 2$ m OT column with fivelayer gold nanoparticles linked with C18. Sixty nanometer gold nanoparticles were self-assembled layer by layer on the inner wall and C18 was then linked on the gold nanoparticles to give the column 
hydrophobic character ${ }^{90}$. The column was used with an in-house made trap column $(50 \mu \mathrm{m} I D \times 1 \mathrm{~cm}$, $5 \mu \mathrm{m}$ C18 particles) to analyze $80 \mathrm{HepG} 2$ cells. In total, 650 proteins were identified in triplicate runs by the nanoLC-MS/MS system.

Aydogan made a $75 \mu \mathrm{m}$ ID OT chiral column by in-situ polymerization of 3-chloro-2hydroxypropylmethacrylate (HPMA-Cl) and ethylene dimethacrylate (EDMA). The reactive chloro groups at the surface of the porous stationary phase were reacted with $\beta$-cyclodextrin $(\beta-C D)^{91}$.

Plate numbers up to 26000 plates/m was achieved. Separation of amino acid enantiomers in a fruit juice sample was demonstrated.

A $75 \mu \mathrm{m}$ ID OT silica-based porous layer micro-cell membrane (mCMC) column was prepared by physical adsorption of rabbit red blood cell (rRBC) membranes onto the inner surface of the OT capillary by Zhang et $\mathrm{al}^{92}$. This $\mathrm{mCMC}$ column may be of great interest for further application developments in biological affinity chromatography (BAC) technology, employing a proteinimmobilized matrix as stationary phase for the investigation of binding interactions between molecules and specific receptors as well as for screening bioactive compounds from complex matrices.

\section{OT capillaries without coating}

Liquid chromatography has also been performed with micelles in OT capillaries ${ }^{93}$. Chromatography was done in 25-200 $\mu \mathrm{m}$ ID and 60 or $120 \mathrm{~cm}$ long fused silica capillaries against a flow of predominantly aqueous surfactant solutions above the critical micelle concentration as mobile phase. However, the authors state that although the green aspects of the proposed OT-LC method are clear (i.e., the complete removal of chemical waste), the peak shapes, analyte sensitivity, and baseline stability were not as good as that obtained with packed column LC.

Narrow (1.5 - $10 \mu \mathrm{m}$ ID) bare silica capillaries have been used for hydrodynamic chromatography separations ${ }^{94-96}$. The hydrodynamic mobility is a function of the ratio between the effective radius of an analyte and the radius of the open capillary. Separation of single molecule DNA has been obtained for the study of DNA conformation ${ }^{94}$ and DNA-biomolecule interactions ${ }^{95}$ using aqueous buffers.

However, Duan et al. performed protein separations in 300 and $500 \mathrm{~nm}$ ID cylindrical self-enclosed nanocapillaries, although they point out that the separation cannot be explained by hydrodynamic chromatography alone ${ }^{97}$. In their paper, they also present normal phase, reversed phase and ionvalence chromatography in the nanochannels, which are integrated in a chip, and also can be classified as belonging to the extended-nano LC category (see below). 
In summary: the open tubular format is still under development, but columns are not commercially available. Despite several significant advances and applications, the OT format continues to be a niche format. Key advantages include high sensitivity and resolution. Key disadvantages include lack of commercial availability and high demands on the operator.

\section{Chip-format}

As an alternative to nanoLC columns in the form of a cylinder formed tube, separations can be done in a channel in a microchip format. The separation channel is typically a ca $50 \mu \mathrm{m} \times 75 \mu \mathrm{m}$ trapezoidal cross-section ${ }^{98}$. The same chromatography as in conventional nanoLC systems can be performed; that is, the separation can be carried out using a packed bed, a monolithic bed or in the open channel format. The main difference is that the microchip format can be a "modular" device with incorporated features allowing "plug and play" for the users, and this format may be operated by individuals with less specialized training. The advantages of microchip LC have led to the commercialization of various formats of microchip LC columns and interfaces with mass spectrometers in the past decade. Several reviews on microchip LC have been published recently showing the interest in this technique ${ }^{99-101}$. Although monolithic beds are rather easily made in microchip channels, particle packed channels are more used.

\section{Commercial columns}

Vendors (e.g. Agilent, Waters, Sciex) offer packed column microchips tailored for proteomics, small molecules, and other applications, as well as custom chips. Typically, a 40-150 mm long analytical column is used in combination with a short trap column, which may be of different chemistry for increased selectivity. Information on the bed width and depth is not always easily obtained; however, $75 \mu \mathrm{m}$ and $85 \mu \mathrm{m}$ appear to be a common bed width in the nano format. Some challenges of pressuredriven chip LC have been reported, and Lotter et al. have studied various approaches to connect pressure-resistant glass chips with HPLC pumps up to 500 bar $^{102}$.

\section{Applications of chip-format columns}

Due to the commercial availability and ease of use, microchip LC-MS has been widely applied, in proteomics ${ }^{103-105}$, but also for other applications ${ }^{106-108}$. However, because the technique is often used as an aid in solving a research problem, the use of chip LC is not necessarily revealed in literature searches. 


\section{New chip-format columns}

The need for specialized equipment for making the chip format, has led to that much of the development is now carried out by companies, and less has been done by individual groups lately.

A glass chip with a $35 \mathrm{~mm}$ long column segment (with width $90 \mu \mathrm{m}$ and depth $40 \mu \mathrm{m}$ packed with C18 BEH 3.5 or $2.5 \mu$ m particles) was used by Heiland et al. who utilized temperature as an active parameter for increased speed and efficiency ${ }^{109}$. They were able to reduce the separation time by more than a half with a thermal gradient from 90 to $180^{\circ} \mathrm{C}$.

Hao et al. have developed a modular microfluidic platform composed of independent pretreatment, LC separation, and nESI chips, for determination of pesticide metabolites and peptides ${ }^{110}$. The lengths of the rectangle regions in pre-treatment and LC separation modules were $2.5 \mathrm{~mm}$ and $20 \mathrm{~mm}$, respectively. Micro-pillar (20 $\mu \mathrm{m}$ diameter) arrays were integrated in both modules to avoid the collapse of channels. Both the trap (enrichment) and LC column (with micro-channels $40 \mu \mathrm{m}$ in depth) were packed with $5 \mu \mathrm{m}$ silica-based particles.

Even though the channels are wider than $100 \mu \mathrm{m}$, the development of the first two-dimensional LCchip, with a heart-cut approach, is included. Two different columns packed with reversed phase and chiral stationary phase material were integrated on a microfluidic glass chip, coupled to $\mathrm{MS}^{111}$. The channels were $155 \mu \mathrm{m}$ wide and $45 \mu \mathrm{m}$ deep, and a C18 $(5 \mu \mathrm{m})$ phase was used in the first dimension and a chiral stationary phase $(5 \mu \mathrm{m})$ in the second dimension.

Heiland et al. used the glass chip (from ${ }^{109}$ ) packed with a chiral stationary phase $(5 \mu \mathrm{m})$ and C18 $(3 \mu \mathrm{m})$, and presented the first combination of microchip-supercritical-fluid chromatography (SFC) for fast and efficient separation of enantiomers and PAHs, respectively ${ }^{112}$.

In summary, the chip format continues to be developed, especially commercially but also noncommercially, and is applied in a variety of contexts. Key advantages include ease of use. Key disadvantages include limited flexibility.

\section{Pillar array columns}

Desmet and co-workers have, based on earlier work by Regnier and co-workers, developed an exciting new format of nanoLC separation columns, which they call micropillar array columns (PACs). Ultra-high efficiencies are obtained with an optimized pillar diameter $(5 \mu \mathrm{m})$ and interpillar distance $(2.5 \mu \mathrm{m})^{113}$. Such columns have been used in combination with MS for peptide mapping of monoclonal antibodies (mAbs) and antibody-drug conjugates (ADCs) ${ }^{4}$. 
Such columns are now commercially available as PharmaFluidics' $\mu \mathrm{PAC}^{\mathrm{TM}}$ columns in the chip format. The perfected, and importantly, reproducible order of the separation bed virtually eliminates axial peak dispersion, resulting in a high efficiency. The freestanding nature of the pillars leads to much lower backpressure allowing the use of very long columns and shorter conditioning. Commercial columns come in length of $50 \mathrm{~cm}$ and $2 \mathrm{~m}$ with $\mathrm{C} 18$ end-capped stationary phase, with a trap column with the same stationary phase, in the cylindrically shaped pillar form. $\mu \mathrm{PAC}^{\mathrm{TM}}$ columns have been shown to provide excellent proteomics capabilities, e.g. consistent identifications of more than 5,000 proteins using 10-hour long gradients ${ }^{114}$ (Figure 4). The only drawback of these columns seems to be the price.

Figure 4 approximately here

Desmet and co-workers have very recently further developed the design of such pillar array columns. They describe the application of a sol-gel procedure on radially elongated pillars (REPs) using tetramethoxysilane and methyltrimethoxysilane, with subsequent octadecylsilylation ${ }^{115}$. An increase in accessible specific surface by a factor of 112 compared to a nonporous REP was observed. Plate heights as low as $0.4-0.8 \mu \mathrm{m}(\mathrm{k}=0-1.97)$ and kinetic plot analysis demonstrated that the column will deliver more theoretical plates per unit of time than a $5 \mu \mathrm{m}$ core shell packed bed when plate number higher than $1.0 \times 10^{4}$ is required. They have followed up this study which focused only on the on-chip performance with a study on attainable performance under practical conditions (i.e. a sufficiently long column in commercial LC hardware with external detection) ${ }^{116}$. Separation of alkylphenones and peptides was studied in a $16.5 \mathrm{~cm}$ long, $1 \mathrm{~mm}$ wide channel (three lanes of $5.5 \mathrm{~cm}$ long channels connected by turns). The minimum plate height of $1.4 \mu \mathrm{m}$ for octanophenone $(\mathrm{k}=2.21)$ observed in isocratic mode was 5 times smaller than the smallest off-column plate height previously reported for porous pillar array columns for a retained component. This advantage is related to the earlier introduced shape of the radially elongated pillar bed that outperforms the cylindrically shaped pillar bed in terms of the plate height.

Furthermore, they have adjusted the preparation conditions to make a 1.2-fold thicker layer on the porous layer REP array column ${ }^{117}$. The mesoporosity of the layer was controlled by changing the hydrothermal treatment temperature from $105^{\circ} \mathrm{C}$ to $80^{\circ} \mathrm{C}$. When performing a $180 \mathrm{~min}$ gradient elution on a $16.5 \mathrm{~cm}$ long column, the peak capacity for an alkylphenone mixture was 315 and 365 for the combination of thin layer and large mesopores, and thick layer and small mesopores, respectively. For peptides, the thicker layer was still favorable, providing a conditional peak capacity of 245 for a commercially available peptide mixture. However, lager mesopores were more advantageous for large 
molecules (> 1000), because of less content of small pores which hinder the diffusion of large molecules in pores in the layer.

In summary: The pillar array format, carefully developed over a number of years for optimizing chromatographic traits, is now commercially available, and has been shown to be a powerful tool in e.g. proteomics. Key advantages include very high chromatographic performance at low pressures. Key disadvantages include high costs for commercial products (as of today).

\section{Extended-nanoLC}

Kitamori and co-workers have developed a technique which they call "extended-nano LC"118. They perform pressure-driven chromatography in channels which are down to $\sim 100 \mathrm{~nm}$ wide and deep. The separation column is an extended-nano fluidic channel which is fabricated on a glass chip. Advantages of extended-nanoLC are the use of extremely small sample volumes, the speed and the high separation efficiencies (plate numbers of up to $\left.1.4 \times 10^{4}\right)^{119}$. In their review paper from 2017 ${ }^{118}$, fundamentals of the extended-nano chromatography technique are summarized, as is the instrumentations used to realize attoliter sample injections and sensitive detection methods. The application of the extended-nanoLC system for analysis of a small sample (39 fL) from a single living human cell has been demonstrated in combination with the femtoliter sampling interface ${ }^{120}$ (Figure 5). Gradient elution is also possible with the extended-nano LC format, which Kitamori and co-workers also call femtoliter LC. Shimizu et al. have developed a flexible gradient system using standard HPLC pumps and an external mixer with a simple sample injection system, and showed its potential for separation of intact proteins ${ }^{121}$. The separation nanochannel size was of $950 \mathrm{~nm}$ depth, $5.0 \mu \mathrm{m}$ width and $10 \mathrm{~mm}$ length in this case, and the inner surface of the channels was modified with octadecylsilyl (ODS) groups.

In summary, although in its infancy, extended-nanoLC is a technological approach that may push the separation technology towards subcellular analysis. Key advantages include a potential for analyzing very small samples. Key disadvantages include a lack of commercial products and high demands to the operator (as of today).

Figure 5 approximately here 


\section{Conclusions}

Even though it has existed for decades, the nanoLC column continues to be developed and be a key tool in a wide range of cutting-edge research areas. The traditional packed column is being refined and is still the most popular choice, especially in the common tube format, but also in the chip format. However, it is beginning to see a contender in the pillar array column, which is now commercially available. There is great enthusiasm for this variant, especially in proteomics environments. Although older than the pillar array column, the monolith and the open tubular variants are prone to being bypassed in popularity, perhaps due to their reputations as being difficult to reproduce or operate. On the other hand, the monolith shows an enormous versatility, and may be applied for a number of challenging applications. However, it is therefore important that column developers demonstrate their innovations with actual samples and "killer applications" rather than relying on standards and simple protein mixtures.

\section{Conflicts of interest}

There are no conflicts of interest to declare.

\section{Acknowledgment}

This work was partially supported by the Research Council of Norway through its Centre of Excellence scheme, project number 262613.

\section{References}

1. S. R. Wilson, T. Vehus, H. S. Berg and E. Lundanes, Bioanalysis, 2015, 7, 1799-1815.

2. L. E. Blue, E. G. Franklin, J. M. Godinho, J. P. Grinias, K. M. Grinias, D. B. Lunn and S. M. Moore, J. Chromatogr. A, 2017, 1523, 17-39.

3. M. V. Novotny, J. Chromatogr. A, 2017, 1523, 3-16.

4. K. Sandra, J. Vandenbussche, I. Vandenheede, B. Claerebout, J. Op de Beeck, P. Jacobs, W. De Malsche, G. Desmet and P. Sandra, LC-GC Eur., 2018, 31, 155-166.

5. M. Dams, J. L. Dores-Sousa, R.-J. Lamers, A. Treumann and S. Eeltink, Chromatographia, 2019, 82, 101-110.

6. A. J. Chetwynd and A. David, Talanta, 2018, 182, 380-390.

7. I. Sierra, M. L. Marina, D. Perez-Quintanilla, S. Morante-Zarcero and M. Silva, Electrophoresis, 2016, 37, 2538-2553.

8. S. Fanali, Electrophoresis, 2017, 38, 1822-1829.

9. K. B. Lynch, A. Chen and S. Liu, Talanta, 2018, 177, 94-103.

10. J. Sestak, D. Moravcova and V. Kahle, J. Chromatogr. A, 2015, 1421, 2-17.

11. M. Schoebinger, O.-J. Klein and G. Mitulovic, Separations, 2016, 3, 6. 
12. M. C. S. Levernaes, O. K. Brandtzaeg, S. F. Amundsen, L. Reubsaet, E. Lundanes, T. G. Halvorsen and S. R. Wilson, Anal. Chem. , 2018, 90, 13860-13866.

13. T. T. Tran, R. C. Bollineni, C. J. Koehler and B. Thiede, Analyst, 2018, 143, 4359-4365.

14. L. Zhang, C.-W. Liu and Q. Zhang, Anal. Chem. , 2018, 90, 1081-1086.

15. Z. Li, J. Tatlay and L. Li, Anal. Chem., 2015, 87, 11468-11474.

16. J. Alcantara-Duran, D. Moreno-Gonzalez, M. Beneito-Cambra and J. F. Garcia-Reyes, Talanta, 2018, 182, 218-224.

17. N. A. Kulak, P. E. Geyer and M. Mann, Mol. Cell. Proteomics, 2017, 16, 694-705.

18. H. S. Berg, K. E. Seterdal, T. Smetop, R. Rozenvalds, O. K. Brandtzaeg, T. Vehus, E. Lundanes and S. R. Wilson, J. Chromatogr. A, 2017, 1498, 111-119.

19. S. E. Spencer, T. N. Corso, J. G. Bollinger, C. M. Henderson, A. N. Hoofnagle and M. J. MacCoss, Anal. Chem., 2017, 89, 2383-2389.

20. F. Rigano, A. Albergamo, D. Sciarrone, M. Beccaria, G. Purcaro and L. Mondello, Anal. Chem. 2016, 88, 4021-4028.

21. G. D'Orazio, R. Kakava, A. Volonterio, S. Fanali and B. Chankvetadze, Electrophoresis, 2017, 38, 1932-1938.

22. X. Shao, X. Wang, S. Guan, H. Lin, G. Yan, M. Gao, C. Deng and X. Zhang, Anal. Chem. , 2018, 90, 14003-14010.

23. Y. Zhu, P. D. Piehowski, R. Zhao, J. Chen, Y. Shen, R. J. Moore, A. K. Shukla, V. A. Petyuk, M. Campbell-Thompson, C. E. Mathews, R. D. Smith, W.-J. Qian and R. T. Kelly, Nat. Commun., 2018, 9, 1-10.

24. N. Tanaka and D. V. McCalley, Anal. Chem. , 2016, 88, 279-298.

25. B. A. Rogers, Z. Wu, B. Wei, X. Zhang, X. Cao, O. Alabi and M. J. Wirth, Anal. Chem., 2015, 87, 2520-2526.

26. L. G. Falkenby, G. Such-Sanmartin, M. R. Larsen, O. Vorm, N. Bache and O. N. Jensen, J. Proteome Res., 2014, 13, 6169-6175.

27. N. Bache, P. E. Geyer, D. B. Bekker-Jensen, O. Hoerning, L. Falkenby, P. V. Treit, S. Doll, I. Paron, J. B. Muller, F. Meier, J. V. Olsen, O. Vorm and M. Mann, Mol. Cell. Proteomics, 2018, 17, 2284-2296.

28. Z. Zajickova, J. Sep. Sci., 2017, 40, 25-48.

29. R. E. Majors, Labcompare, 2018.

30. T. Vehus, K. E. Seterdal, E. Lundanes, S. R. Wilson and S. Krauss, Future Sci OA, 2016, 2, FSO119.

31. L. Kucera, S. Fanali, Z. Aturki, T. Pospisil and P. Bednar, J. Chromatogr. A, 2016, 1428, 126133.

32. O. K. Brandtzaeg, B.-T. Roeen, S. Enger, E. Lundanes and S. R. Wilson, Anal. Chem. , 2017, 89, 8667-8673.

33. C. Aydogan, A. Goekaltun, A. Denizli and Z. El-Rassi, J. Sep. Sci., 2019, 42, 962-979.

34. T. Hong, X. Yang, Y. Xu and Y. Ji, Anal. Chim. Acta, 2016, 931, 1-24.

35. X. Ding, J. Yang and Y. Dong, J. Pharm. Anal., 2018, 8, 75-85.

36. F. Maya and B. Paull, J. Sep. Sci., 2019, 42, 1564-1576.

37. J. L. Dores-Sousa, A. Fernandez-Pumarega, J. De Vos, M. Laemmerhofer, G. Desmet and S. Eeltink, J. Sep. Sci., 2019, 42, 522-533.

38. F. Svec, Electrophoresis, 2017, 38, 2810-2820.

39. J. Urban, J. Sep. Sci., 2016, 39, 51-68.

40. I. Nischang and T. J. Causon, TrAC, Trends Anal. Chem., 2016, 75, 108-117.

41. Z. Liu, J. Ou and H. Zou, TrAC, Trends Anal. Chem., 2016, 82, 89-99.

42. P.-L. Tsai, T.-Y. Sung, C.-Y. Chong, S.-Y. Huang and S.-F. Chen, Anal. Methods, 2018, 10, 47564764.

43. Z. Zhang, J. Xu, D. Hussain and Y.-Q. Feng, J. Chromatogr. A, 2016, 1453, 71-77.

44. X. Wang, A. Lamprou, F. Svec, Y. Bai and H. Liu, J. Sep. Sci., 2016, 39, 4544-4548. 
45. D. Xu, H. Shao, R. Luo, Q. Wang, E. Sanchez-Lopez, S. Fanali, M. L. Marina and Z. Jiang, J. Chromatogr. A, 2018, 1557, 43-50.

46. C. Aydogan, Anal. Bioanal. Chem., 2016, 408, 8457-8466.

47. T. Hara, H. Kobayashi, T. Ikegami, K. Nakanishi and N. Tanaka, Anal. Chem., 2006, 78, 76327642.

48. T. Hara, G. Desmet, G. V. Baron, H. Minakuchi and S. Eeltink, J. Chromatogr. A, 2016, 1442, 42-52.

49. T. Hara, S. Eeltink and G. Desmet, J. Chromatogr. A, 2016, 1446, 164-169.

50. T. Hara, S. Futagami, W. De Malsche, S. Eeltink, H. Terryn, G. V. Baron and G. Desmet, Anal. Chem. , 2017, 89, 10948-10956.

51. H. Kobayashi, M. Sukegawa, K. Fujimura, T. Kubo and K. Otsuka, Chromatography, 2016, 37, 133-139.

52. C. Wu, Y. Liang, K. Yang, Y. Min, Z. Liang, L. Zhang and Y. Zhang, Anal. Chem. , 2016, 88, 15211525.

53. X. Lv, W. Tan, Y. Chen, Y. Chen, M. Ma, B. Chen and S. Yao, J. Chromatogr. A, 2016, 1454, 4957.

54. E.-D. Racha, P. Gay, V. Dugas and C. Demesmay, J. Sep. Sci., 2016, 39, 842-850.

55. H. Lyu, H. Zhao, W. Qin and Z. Xie, J. Sep. Sci., 2017, 40, 4521-4529.

56. H. Wang, W. Hu, Q. Zheng, W. Bian and Z. Lin, J. Sep. Sci., 2017, 40, 2344-2354.

57. H. Zhang, J. Ou, Y. Wei, H. Wang, Z. Liu and H. Zou, J. Chromatogr. A, 2016, 1440, 66-73.

58. H. Zhang, S. Ma, Y. Yao, Y. Li, Y. Li, J. Ou, M. Ye and Y. Wei, J. Chromatogr. A, 2017, 1524, 135142.

59. C. Demir, C. Kip and A. Tuncel, Electrophoresis, 2018, 39, 2919-2928.

60. J. Su, L. Yang and Q. Wang, J. Chromatogr. A, 2018, 1533, 136-142.

61. K. Szwed, J. Ou, G. Huang, H. Lin, Z. Liu, H. Wang and H. Zou, J. Sep. Sci., 2016, 39, 1110-1117.

62. M. Wolter and M. Laemmerhofer, J. Chromatogr. A, 2017, 1497, 172-179.

63. D. Xu, Q. Wang, E. Sanchez-Lopez, Z. Jiang and M. L. Marina, J. Chromatogr. A, 2019, 1593, 63-72.

64. S. Ma, Y. Wang, H. Zhang, Y. Li, J. Ou, Y. Wei and M. Ye, Talanta, 2019, 198, 432-439.

65. S. Liu, J. Peng, Z. Liu, Z. Liu, H. Zhang and R. a. Wu, Sci. Rep., 2016, 6, 34718.

66. S. Liu, J. Peng, H. Zhang, X. Li, Z. Liu, X. Kang, M. Wu and R.-a. Wu, J. Chromatogr. A, 2017, 1498, 64-71.

67. C. Brede, B. Hop, K. Joergensen and O. Skadberg, Scand. J. Clin. Lab. Invest., 2016, 76, 195201.

68. K. B. Lynch, J. Ren, M. A. Beckner, C. He and S. Liu, Anal. Chim. Acta, 2019, 1046, 48-68.

69. S. Shibasaki, M. Karasaki, W. Aoki and M. Ueda, Proteomes, 2018, 6.

70. D. Ishii and T. Takeuchi, J. Chromatogr. Sci., 1980, 18, 462-472.

71. G. Yue, Q. Luo, J. Zhang, S.-L. Wu and B. L. Karger, Anal. Chem., 2007, 79, 938-946.

72. H. K. Hustoft, O. K. Brandtzaeg, D. Misaghian, T. Greibrokk, S. R. Wilson, E. Lundanes, M. Rogeberg, S. B. Torsetnes and L. Reubsaet, Sci Rep, 2013, 3, 3511.

73. H. K. Hustoft, T. Vehus, O. K. Brandtzaeg, S. Krauss, T. Greibrokk, S. R. Wilson and E. Lundanes, PLoS One, 2014, 9, e106881/106881-e106881/106810, 106810 pp.

74. S. C. Lam, E. Sanz Rodriguez, P. Haddad and B. Paull, Analyst, 2019, 144, 3464-3482.

75. O. Skjaervoe, O. K. Brandtzaeg, K. B. Lausund, O. Pabst, O. G. Martinsen, E. Lundanes and S. R. Wilson, J. Chromatogr. A, 2018, 1534, 195-200.

76. T. Vehus, H. Roberg-Larsen, J. Waaler, S. Aslaksen, S. Krauss, S. R. Wilson and E. Lundanes, Sci. Rep., 2016, 6, 37507.

77. T. Hara, S. Futagami, S. Eeltink, W. De Malsche, G. V. Baron and G. Desmet, Anal. Chem. , 2016, 88, 10158-10166.

78. T. Hara, S. Futagami, W. De Malsche, G. V. Baron and G. Desmet, J. Chromatogr. A, 2018, 1552, 87-91. 
79. T. Hara, Y. Izumi, M. Nakao, K. Hata, G. V. Baron, T. Bamba and G. Desmet, J. Chromatogr. A, 2018, 1580, 63-71.

80. H. Chen, Y. Yang, Z. Qiao, P. Xiang, J. Ren, Y. Meng, K. Zhang, J. Juan Lu and S. Liu, Analyst, 2018, 143, 2008-2011.

81. Y. Yang, H. Chen, M. A. Beckner, P. Xiang, J. J. Lu, C. Cao and S. Liu, Anal. Chem., 2018, 90, 10676-10680.

82. R. Li, Y. Shao, Y. Yu, X. Wang and G. Guo, Chem. Commun. (Cambridge, U. K.), 2017, 53, 41044107.

83. A. A. Kazarian, E. Sanz Rodriguez, J. A. Deverell, J. McCord, D. C. Muddiman and B. Paull, Anal. Chim. Acta, 2016, 905, 1-7.

84. E. S. Rodriguez, S. C. Lam, P. R. Haddad and B. Paull, Chromatographia, 2019, 82, 197-209.

85. M. Ribeiro da Silva, O. K. Brandtzaeg, T. Vehus, F. M. Lancas, S. R. Wilson and E. Lundanes, J. Chromatogr. A, 2017, 1518, 104-110.

86. L. Peng, M. Zhu, L. Zhang, H. Liu and W. Zhang, J. Sep. Sci., 2016, 39, 3736-3744.

87. H. Wang, Y. Yao, Y. Li, S. Ma, X. Peng, J. Ou and M. Ye, Anal. Chim. Acta, 2017, 979, 58-65.

88. K. Chen, L. Zhang and W. Zhang, J. Sep. Sci., 2018, 41, 2347-2353.

89. M. Zhu, L. Zhang, Z. Chu, S. Wang, K. Chen, W. Zhang and F. Liu, Talanta, 2018, 184, 29-34.

90. X. Shao and X. Zhang, Proteomics, 2017, 17.

91. C. Aydogan, Chirality, 2018, 30, 1144-1149.

92. F. Zhang, X. Zhao, B. Xu, S. Cheng, C. Tang, H. Duan, X. Xiao, W. Du and L. Xu, Anal. Bioanal. Chem., 2016, 408, 2441-2448.

93. J. P. Quirino and F. M. Tarongoy, Green Chem., 2018, 20, 2486-2493.

94. S. M. Friedrich, K. J. Liu and T.-H. Wang, J. Am. Chem. Soc., 2016, 138, 319-327.

95. S. M. Friedrich, R. Bang, A. Li and T.-H. Wang, Anal. Chem., 2019, 91, 2822-2830.

96. X. Wang, C. Cheng, S. Wang, M. Zhao, P. K. Dasgupta and S. Liu, Anal. Chem., 2009, 81, 74287435.

97. L. Duan, Z. Cao and L. Yobas, Anal. Chem., 2016, 88, 11601-11608.

98. S. Ehlert, L. Trojer, M. Vollmer, T. van de Goor and U. Tallarek, J. Mass Spectrom., 2010, 45, 313-320.

99. X. Yuan and R. D. Oleschuk, Anal. Chem., 2018, 90, 283-301.

100. A. Kecskemeti and A. Gaspar, Anal. Chim. Acta, 2018, 1021, 1-19.

101. F. Haghighi, Z. Talebpour and A. S. Nezhad, TrAC, Trends Anal. Chem., 2018, 105, 302-337.

102. C. Lotter, J. J. Heiland, V. Stein, M. Klimkait, M. Queisser and D. Belder, Anal. Chem., 2016, 88, 7481-7486.

103. C. Lammi, G. Aiello, G. Vistoli, C. Zanoni, A. Arnoldi, Y. Sambuy, S. Ferruzza and G. Ranaldi, J. Funct. Foods, 2016, 24, 297-306.

104. Y. Wang, D. Park, A. G. Galermo, D. Gao, H. Liu and C. B. Lebrilla, Proteomics, 2016, 16, 29772988.

105. C. Phoomak, A. Silsirivanit, D. Park, K. Sawanyawisuth, K. Vaeteewoottacharn, C. Wongkham, E. W. F. Lam, C. Pairojkul, C. B. Lebrilla and S. Wongkham, Oncogene, 2018, 37, 5648-5665.

106. D. P. Bishop, L. Blanes, A. B. Wilson, T. Wilbanks, K. Killeen, R. Grimm, R. Wenzel, D. Major, M. Macka, D. Clarke, R. Schmid, N. Cole and P. A. Doble, J. Chromatogr. A, 2017, 1497, 64-69.

107. M.-N. Li, C.-R. Li, W. Gao, P. Li and H. Yang, Anal. Chim. Acta, 2017, 982, 156-167.

108. L. D. Wu, L. R. Ruhaak and C. B. Lebrilla, Methods Mol. Biol., 2017, 1503, 121-129.

109. J. J. Heiland, C. Lotter, V. Stein, L. Mauritz and D. Belder, Anal. Chem., 2017, 89, 3266-3271.

110. Y. Hao, Y. Bao, X. Huang, Y. Hu and B. Xiong, RSC Adv., 2018, 8, 39811-39817.

111. C. Lotter, E. Poehler, J. J. Heiland, L. Mauritz and D. Belder, Lab Chip, 2016, 16, 4648-4652.

112. J. J. Heiland, D. Geissler, S. K. Piendl, R. Warias and D. Belder, Anal. Chem., 2019, 91, 61346140.

113. W. De Malsche, J. Op De Beeck, S. De Bruyne, H. Gardeniers and G. Desmet, Anal. Chem. , 2012, 84, 1214-1219. 
114. J. O. De Beeck, J. Pauwels, N. Van Landuyt, P. Jacobs, W. De Malsche, G. Desmet, A. Argentini, A. Staes, L. Martens, F. Impens and K. Gevaert, bioRxiv, 2019, DOI: 10.1101/472134, 472134.

115. S. Futagami, T. Hara, H. Ottevaere, G. V. Baron, G. Desmet and W. De Malsche, J. Chromatogr. A, 2017, 1523, 234-241.

116. S. Futagami, T. Hara, H. Ottevaere, H. Terryn, G. V. Baron, G. Desmet and W. De Malsche, Analyst, 2019, 144, 1809-1817.

117. S. Futagami, T. Hara, H. Ottevaere, H. Terryn, G. V. Baron, G. Desmet and W. De Malsche, J. Chromatogr. A, 2019, 1595, 58-65.

118. H. Shimizu, A. Smirnova, K. Mawatari and T. Kitamori, J. Chromatogr. A, 2017, 1490, 11-20.

119. H. Shimizu, K. Morikawa, Y. Liu, A. Smirnova, K. Mawatari and T. Kitamori, Analyst, 2016, 141, 6068-6072.

120. L. Lin, K. Mawatari, K. Morikawa, Y. Pihosh, A. Yoshizaki and T. Kitamori, Analyst, 2017, 142, 1689-1696.

121. H. Shimizu, K. Toyoda, K. Mawatari, S. Terabe and T. Kitamori, Anal. Chem., 2019, 91, 30093014. 


\section{Figure legends}

Figure 1: Chromatogram of digested proteins from ten HeLa cells by nano-LC-MS/MS. Reproduced from ref. 22 "Integrated Proteome Analysis Device for Fast Single-Cell Protein Profiling" with permission from American Chemical Society, Copyright 2018.

Figure 2: Spider fractionation (A-C) applied to HeLa digest to obtain elution profiles of every eight fraction (D) and combined fraction (E) showed that $75 \%$ of the total mass of the peptides were concentrated in mainly one or two fractions (F). Reproduced from ref. 17 with permission through CC-BY licensed publication.

Figure 3. Detection of ricin signature peptides using monolithic nanocolumns. Protein digestion was performed online using a multichannel immobilized enzyme reactor. Reproduced from ref. 32 "Multichannel Open Tubular Enzyme Reactor Online Coupled with Mass Spectrometry for Detecting Ricin" with permission from American Chemical Society, Copyright 2017.

Figure 4. Top: Illustration of pillar array columns and set-up. Bottom: comparisons of proteomics performances with standard packed nanoLC column format Reproduced from ref. 114 with permission through CC-BY licensed publication.

Figure 5: Single cell sampling interface device for femtoliter sampling using an extended-nano channel with 10-100 nm width. Reproduced from ref. 120 with permission from Royal Chemical Society, Copyright 2017. 
(Intensity $10^{6}$ )

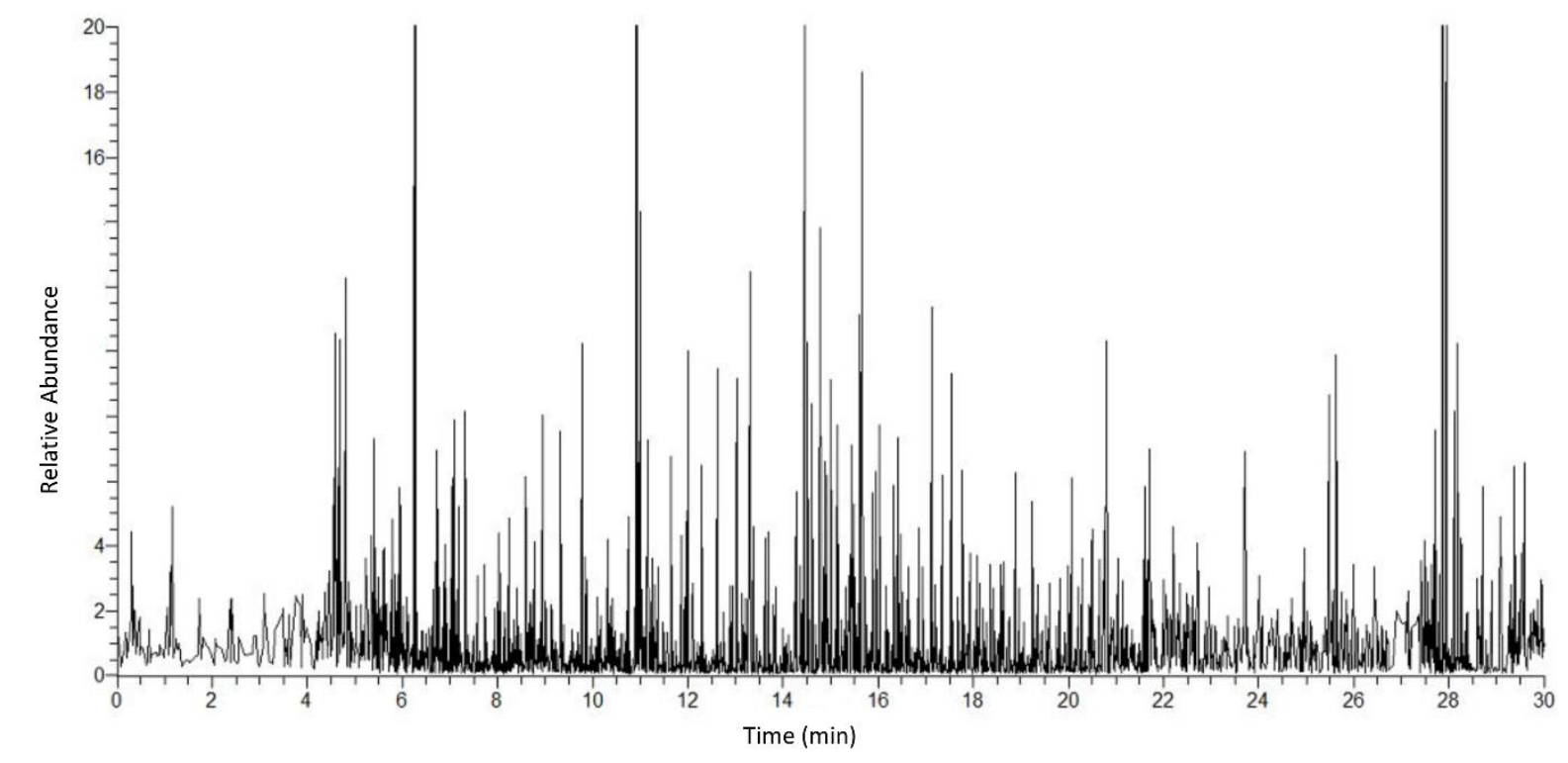

Figure 1
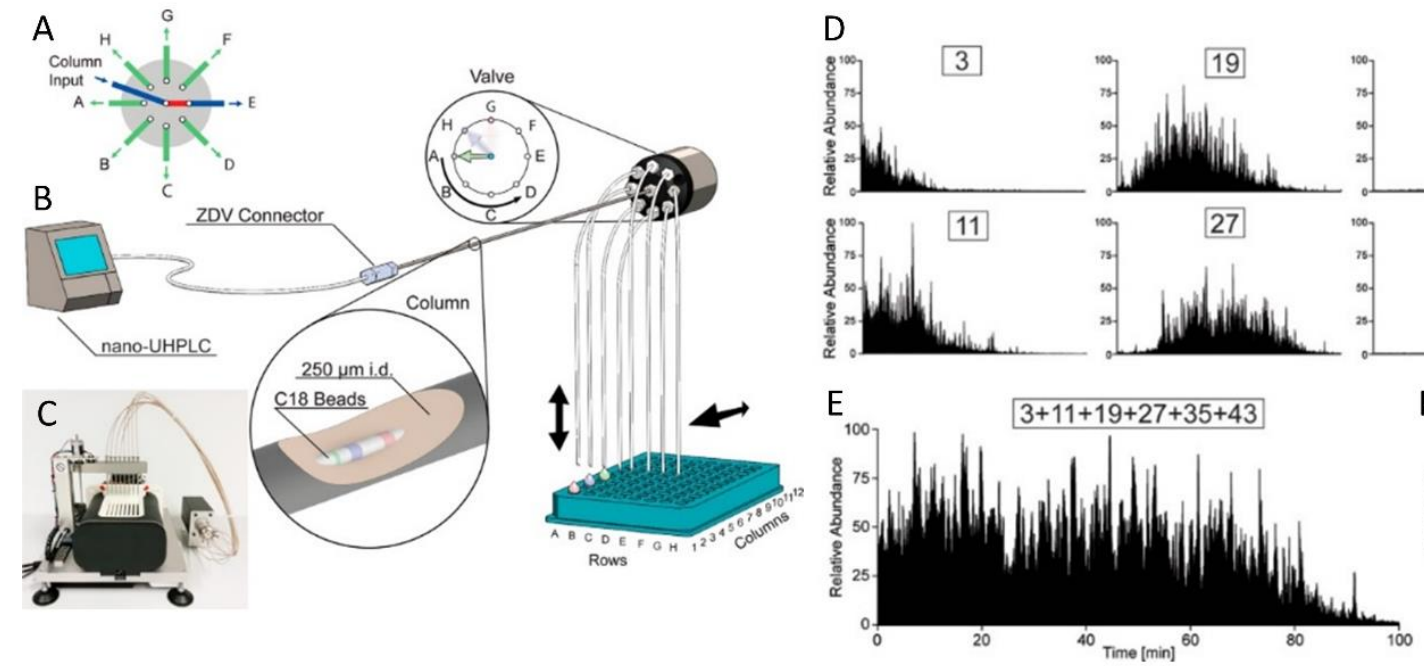

35

43 


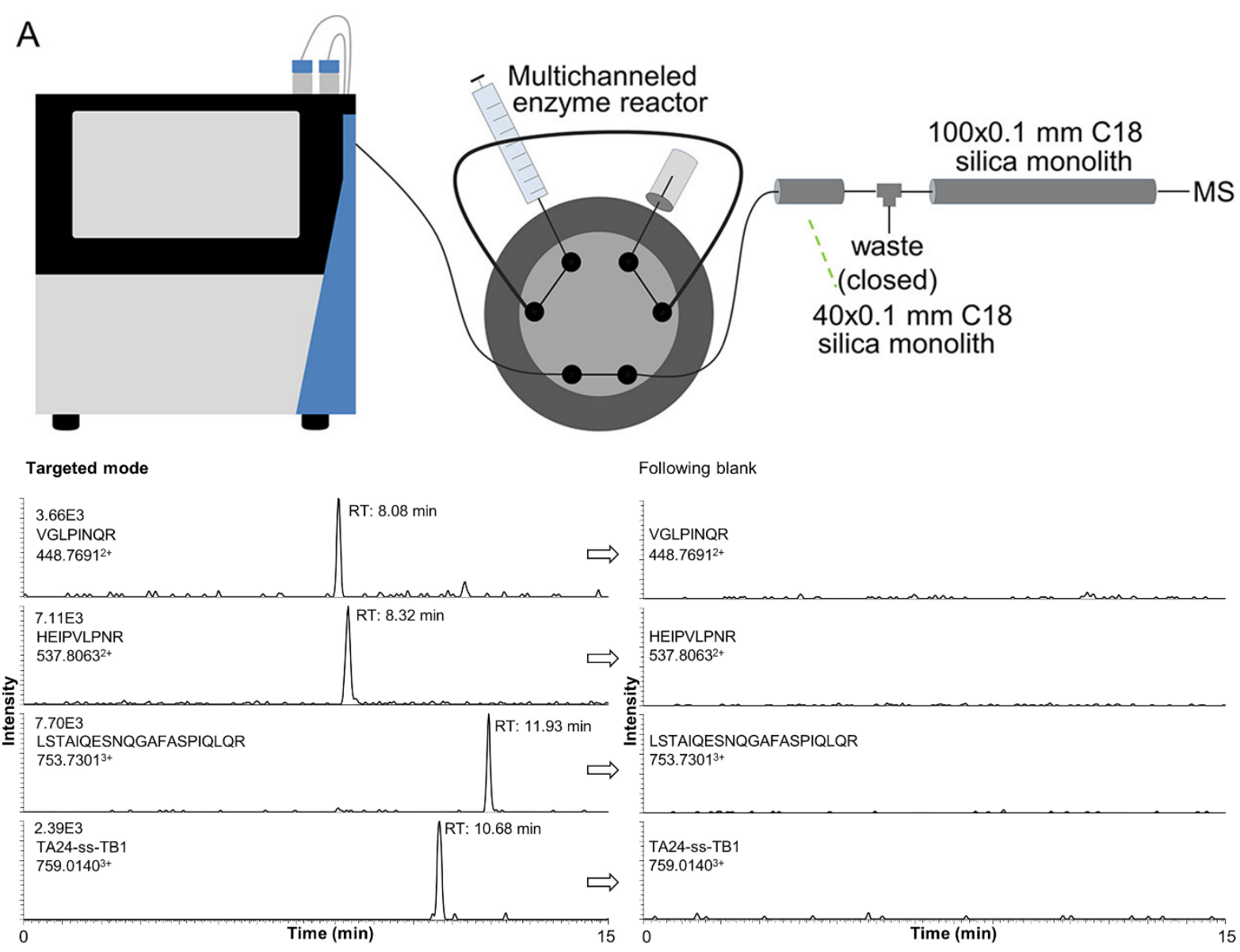

Figure 3

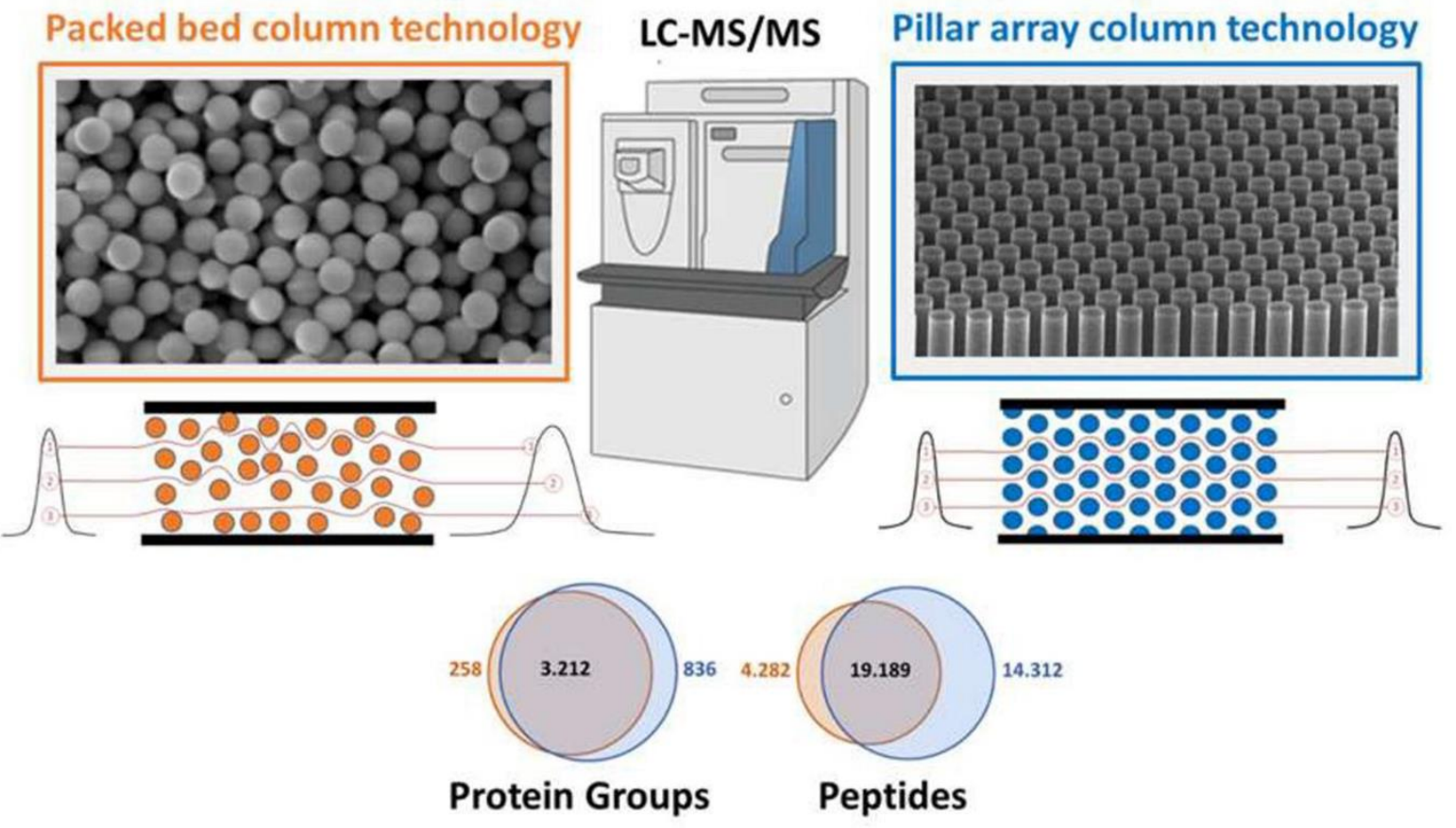

Figure 4 


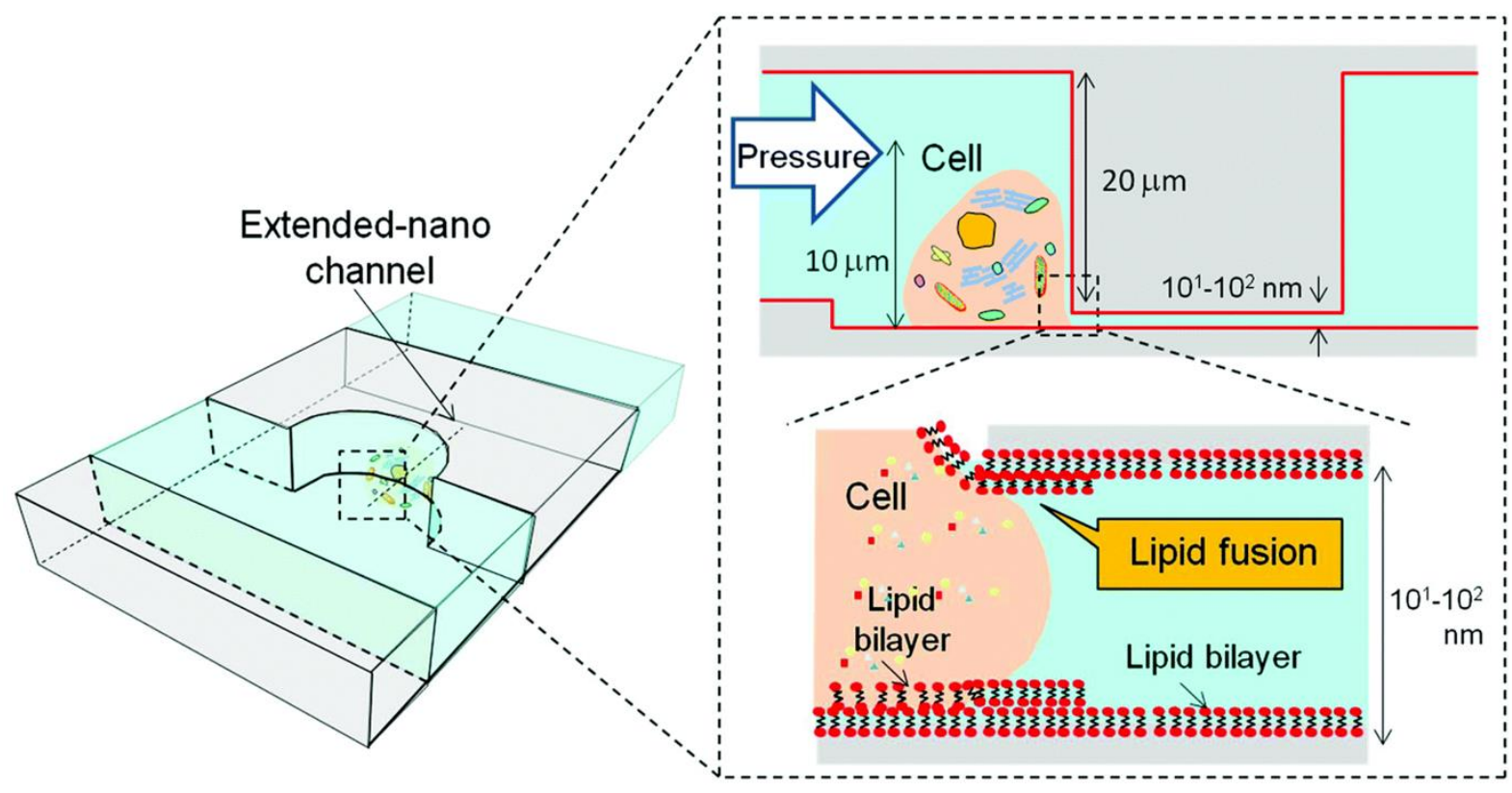

Figure 5 